\title{
CORRIGENDUM
}

\section{Sexuality in patients with spinal cord injuries due to attempted suicide}

G Lombardi

Spinal Cord (2008) 46, 826; doi:10.1038/sc.2008.26

Correction to: Spinal Cord 46, 53-57; advance online publication, 10 April 2007; doi:10.1038/sj.sc.3102062

Since the publication of the above article, the authors have noticed that the author name G Del Popolo was published incorrectly in the above-referenced paper. The correct author list is shown below:

Lombardi G, Mondaini N, Iazzetta P, Macchiarella A, Del Popolo G.

The authors would like to apologize for this error. 